\title{
Introduction of an open source middleware for automatic FOV adjustment in interactive MRI according to a medical tracking-system
}

Felix Güttler ${ }^{1}$, Peter Krauß ${ }^{2}$, Jonathan Guntermann ${ }^{2}$, Andreas Heinrich ${ }^{1}$, Ulf Teichgräber ${ }^{1},{ }^{1}$ Department of Radiology, University Hospital Jena, Jena, Germany, ${ }^{2}$ Department of Radiology, Charité, Berlin, Germany, felix.guettler@med.uni-jena.de

\section{Introduction}

In real-time MRI-guided interventions and surgery [1], the field-of-view (FOV) of interactive sequences needs to be permanently changed according to the instruments movement. Dynamic tracking [2] allows the automatic adaption of the FOV to the instruments location acquired by a medical tracking-system. To accomplish manufacturer independent use of dynamic tracking, a middleware was developed allowing the use of various MRIs and tracking-systems.

\section{Methods}

The middleware provides unified navigation services for various tracking-systems (i.e. NDI Polaris) and MRI scanners (i.e. Philips HFO). Positioning data from target objects is transformed to MRI specific matrices. The MRI adjusts the FOV accordingly and acquires an image, which is returned to the middleware. The use of the system was evaluated by a specific implementation of a biopsy assistance navigation software. To simplify navigation, the target slice and needle position were represented in VR, which can be referenced independent from MR-image quality. Synchronization between the tracking-system and the MRI was measured by biopsy simulations $(n=100)$ performed on a phantom. Attempts were carried out by five interventionalists and five untrained probands. Time and hit-rate were compared to reference values [3].

\section{Results}

The implemented middleware creates a link between the MRI and the tracking device and reliably aligned the FOV on the puncture needle. No connections were dropped during the experiment. The representation of the image plane and of the current FOV allows a simplified navigation, results in an efficient decrease of procedure time by $22 \%$. The suggested corrections enable also the untrained group to reach a slightly higher hit rate and reduction of interventional time.

\section{Conclusion}

The middleware allows the implementation of specific navigation applications without precise knowledge of the underlying manufacturer specific hard- or software. In the experimental setup the middleware allowed the image plane of the dynamic MR-sequence to always show the monitored instrument, guided by the coordinates of a tracking-system.

\section{References}

1. Papanikolaou, I.S., et al., Percutaneous transhepatic cholangiodrainage under real-time MRI guidance: Initial experience in an animal model. Digestive and liver disease: official journal of the Italian Society of Gastroenterology and the Italian Association for the Study of the Liver, 2011.

2. Rump, J.C., et al., The impact of imaging speed of MR-guided punctures and interventions in static organs--A phantom study. European journal of radiology, 2011.

3. F.V. Guettler, M. de Bucourt, P. Krauss, J. Guntermann, J. Rump, A. Heinrich and U. Teichgraeber, "Dynamic Tracking on the Example of MR Imaging-guided Facet Joint Infiltration", Scientific Informal Presentations of the RSNA 2011 Annual Meeting, Nov. 2011.

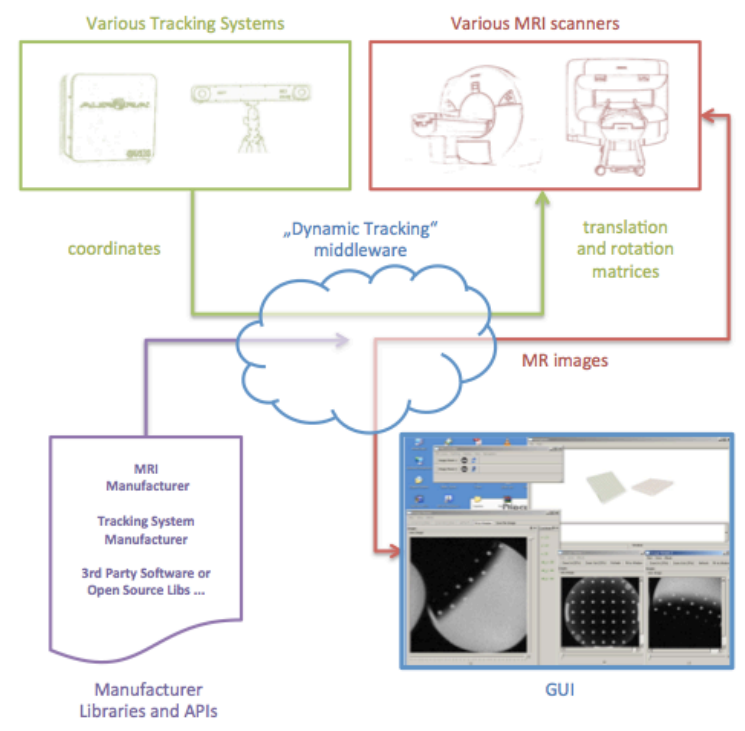

